\title{
Wissenskultur als Erfolgsfaktor für ein ganzheitliches Wissensmanagement
}

\section{Ergebnisse aus einer Fallstudie in der Schweizerischen Post}

Führung, ganzheitliches Wissensmanagement, öffentlicher Sektor, Postsektor, Produktivität, Unternehmenskultur, Wettbewerb, Wissenskultur

Der bewusste und zielgerichtete Umgang mit Wissen gewinnt in einer durch weit reichende technologische und soziale Veränderungen geprägten Welt immer mehr an Bedeutung. In einem durch großen Konkurrenzdruck bestimmten Markt können nur diejenigen Unternehmen überleben, die ihr Wissenspotenzial systematisch ausschöpfen und von einem ganzheitlichen Wissensmanagement ausgehen. Hierbei spielen jedoch weniger die Technologie, sondern vielmehr die Unternehmenskultur bzw. die Wissenskultur und der Mensch eine erfolgversprechende Rolle. Obwohl dieser Wirkungszusammenhang schon häufiger vermutet wurde, bestehen bis heute kaum Literaturbeiträge und wissenschaftliche Untersuchungen zu dieser Thematik. Im vorliegenden Aufsatz wird die Wissenskultur konzeptionell hergeleitet und deren Bedeutung anhand empirischer Ergebnisse aus einer Intensivfallstudie in der Schweizerischen Post aufgezeigt. Weiter werden ausgewählte Maßnahmen zur Entwicklung einer Wissenskultur erläutert. ${ }^{1}$

\section{Einleitung}

Die Ressource „Wissen“ bildet in der heutigen Gesellschaft eine entscheidende Quelle zur Schaffung nachhaltiger Wettbewerbsvorteile für Unternehmen, Sektoren, Regionen sowie ganzer Länder. ${ }^{2}$ Der öffentliche Sektor ist wie der private Sektor gleichermaßen von den Herausforderungen der Wissensgesellschaft ${ }^{3}$ betroffen. So wandelt sich der öffentliche Bereich vermehrt zu einem Dienstleister für Bürger und Unternehmen und muss daher der wachsenden Dynamik und zunehmenden Komplexität des wirtschaftlichen und

1 Der vorliegende Aufsatz basiert auf einem gleichnamigen Forschungsprojekt am Institut für Organisation und Personal der Universität Bern (Schweiz). Die Ergebnisse werden 2006 im Haupt-Verlag (Bern, Stuttgart, Wien) unter dem Titel „Wissenskultur - Erfolgsfaktor für ein ganzheitliches Wissensmanagement“ publiziert. Für weitere Informationen vgl. www.iop.unibe.ch.

2 Vgl. Thom/Harasymowicz-Birnbach (2003a), S. 16.

3 Die Wissensgesellschaft wird wie folgt definiert: „The knowledge economy encompasses all jobs, companies, and industries in which the knowledge and capabilities of people, rather than the capabilities of machines or technologies, determines competitive advantage." Lengnick-Hall/Lengnick-Hall (2003), S. 16. Auch die Wissensgesellschaft ist zwar aufgrund der riesigen Dynamik auf dem Gebiet der Informations- und Kommunikationstechnologie eine technikbasierte Gesellschaft, gewichtet aber im Gegensatz zur Informationsgesellschaft den Menschen, seine Fähigkeiten und Wertvorstellungen stärker. Vgl. Mandl/Reinmann-Rothmeier (2000), S. $6 \mathrm{f}$. 
politischen Umfeldes immer mehr Rechnung tragen. Entsprechend gewinnt das gezielte Management von Wissen auch im öffentlichen Sektor laufend an Bedeutung. ${ }^{4}$ Nach Milner spielt Wissensmanagement für den öffentlichen Sektor eine zentrale Rolle: „To ignore the potential for harnessing and capitalising upon knowledge assets in a public service context is, therefore, to waste opportunities". 5

Entscheidend für den Erfolg von Wissensmanagement ist jedoch die Berücksichtigung der Besonderheiten des öffentlichen Sektors. „However, its success or failure will depend on how KM is adapted to the context of the public sector". ${ }^{6}$ Eine besondere Herausforderung für die erfolgreiche Einführung und Etablierung von Wissensmanagement bildet im öffentlichen Sektor die Überwindung kultureller Barrieren, die eine auf Zusammenarbeit und Verbesserung ausgerichtete Unternehmenskultur erheblich erschweren. ${ }^{7}$ In der Kultur des öffentlichen Sektors werden Misserfolge oder Fehlschläge oftmals nicht toleriert und damit die Entwicklung von Innovationen und neuem Wissen nahezu verunmöglicht. ${ }^{8}$ Damit Wissensmanagement überhaupt Erfolg haben kann, muss unter den Mitarbeitern die Kommunikation, die Bildung von bereichsübergreifenden Netzwerken sowie die Kooperation gefördert und damit längerfristig eine wissensfreundliche Unternehmenskultur aufgebaut werden. ${ }^{9}$

Diese Bedingungen gelten insbesondere für den Postsektor. Traditionelle Postunternehmen mit ihren oftmals starren Strukturen und der relativ langsamen Anpassungsfähigkeit an die Marktbedürfnisse sind im liberalisierten Markt nicht wettbewerbsfähig. Innovation und Modernisierung sind entscheidend und müssen zu Verbesserungen der Qualität und Effizienz führen. Zudem wandeln sich postalische Unternehmen immer mehr von reinen logistischen Unternehmen zu Anbietern von logistischen Dienstleistungen. Wissen wird in diesem Zusammenhang zu einer wichtigen Wettbewerbsressource für die Logistikbranche. Dennoch erhält Wissensmanagement im postalischen Markt bis zum heutigen Zeitpunkt nicht die angemessene Wertschätzung. Aufgrund seiner unterstützenden Funktion für die Entwicklung und Umsetzung von Innovationen erlangt ein zielgerichtetes Wissensmanagement jedoch zunehmend eine zentrale Bedeutung. ${ }^{10}$ "It is therefore of the greatest importance to bring $\mathrm{KM}$ onto the postal scene in order to deliver on innovation in terms of management of product development, communication- and marketing information processes". ${ }^{11}$

4 Vgl. Thom/Harasymowicz-Birnbach (2003b), S. 50 f.; Meyer (2003), S. 11; Wiig (2002), S. 224 f.

5 Milner (2000), S. 69.

6 Cong/Kaushik (2003), S. 28.

7 Vgl. Milner (2000), S. 76; vgl. Nagel/Müller (1999), S. 6 ff.

8 Vgl. Thom/Ritz (2004), S. 142.

9 Vgl. Milner (2000), S. 77.

10 Vgl. Boersma/Banwarie (2004), o. S.; Friedli (2003), S. 148;. Thom/Ritz (2004), S. 21.

11 Boersma/Banwarie (2004), o. S. 


\section{Wissensmanagement}

Wissensmanagement an sich ist nichts Neues. Schon seit Jahrhunderten werden Kenntnisse und Wissen weitergegeben sowie Erfahrungen und Know-how ausgetauscht. ${ }^{12}$ „However, although the term [knowledge management] may be new and trendy, the concept is as old as the pyramids of Giza [Ergänzung durch die Verfasser]". ${ }^{13}$ Auch in Unternehmen fand Wissensmanagement in Form von Netzwerken auf ungeplante und informelle Weise immer schon statt. ${ }^{14}$ Die Thematik hat jedoch erst seit den frühen 90er Jahren große Bedeutung und beachtliche Aufmerksamkeit erhalten. ${ }^{15}$ Das vorhandene informale Wissensmanagement soll mit Hilfe verschiedener Instrumente und Methoden systematisch unterstützt und dadurch formal sowie effizienter gestaltet werden. ${ }^{16}$ So hat Wissensmanagement als Disziplin kaum neue Instrumente entwickelt, sondern zeichnet sich vielmehr durch die Ausschöpfung, aber auch durch die Nutzung von Synergien zwischen wissensmanagementverwandten Disziplinen sowie durch deren Instrumente und Methoden aus. Die im Wissensmanagement verwendeten Ansätze und Instrumente stammen primär von etablierten Wissenschaftsdisziplinen wie Kommunikationswissenschaft, Organisationspsychologie, Soziologie sowie Informations- und Kommunikationstechnologie. ${ }^{17}$

Erfahrungen aus zahlreichen Wissensmanagementprojekten zeigen, dass der Erfolg des Wissensmanagements von mehreren Dimensionen abhängt und nur ein holistischer Ansatz der Komplexität des Themas gerecht wird. ${ }^{18}$ In einem ganzheitlichen Wissensmanagement werden Mensch, Organisation sowie Informations- und Kommunikationstechnologie berücksichtigt (vgl. Abbildung 1). ${ }^{19}$ Dabei ist innerhalb der drei Gestaltungsfelder der Fokus insbesondere auf die Mitarbeiter zu richten. „If we have learned nothing else in four years of observing the knowledge management vanguard, we have seen clearly the importance of getting the approximately $50 / 25 / 25$ people/process/technology balance right from the outset". ${ }^{20}$

12 Vgl. Gamble/Blackwell (2001), S. 2.

13 Lengnick-Hall/Lengnick-Hall (2003), S. 77.

14 Vgl. Birkinshaw (2001), S. 15.

15 Vgl. Hansen u.a. (1999), S. 106; Stenmark (2001), S. 10; Birkinshaw (2001), S. 11.

16 Vgl. Gamble/Blackwell (2001), S. 39.

17 Vgl. Gamble/Blackwell (2001), S. 141.

18 Vgl. Sager/Aebi (2003), S. 105; Adelsberger u.a. (2002), S. 529 ff.; Pawlowsky (2002), S. 112 f.; Alex u.a. (2002), S. 47; Pfeifer u.a. (2001), S. 35; Nohr (2001), S. 6; Pan/Scarbrough (1998), S. 66; Teschke u.a. (2002), S 41; Lee/Choi (2003), S. 181.

19 Vgl. Rüstmann/Thommen (2002), S. 481; Armutat u.a. (2002), S. 20; Pfeifer u.a. (2001), S. 35; Rosenstiel (2000), S. 154; Pieler (2001), S. 124.

20 Ruggles (1998), S. 88; vgl. Hopfenbeck u.a. (2001), S. 267 f. Vom selben Verhältnis gehen auch Lenk und Wengelowski (2002), S. 160, aus: „Ansatzpunkte für Wissensmanagement im öffentlichen Sektor müssen wie im privaten Sektor - davon ausgehen, dass Wissensmanagement ,50\% people, 25\% process, $25 \%$ technology' ist.“; vgl. Augustin (2000), S. 160. 


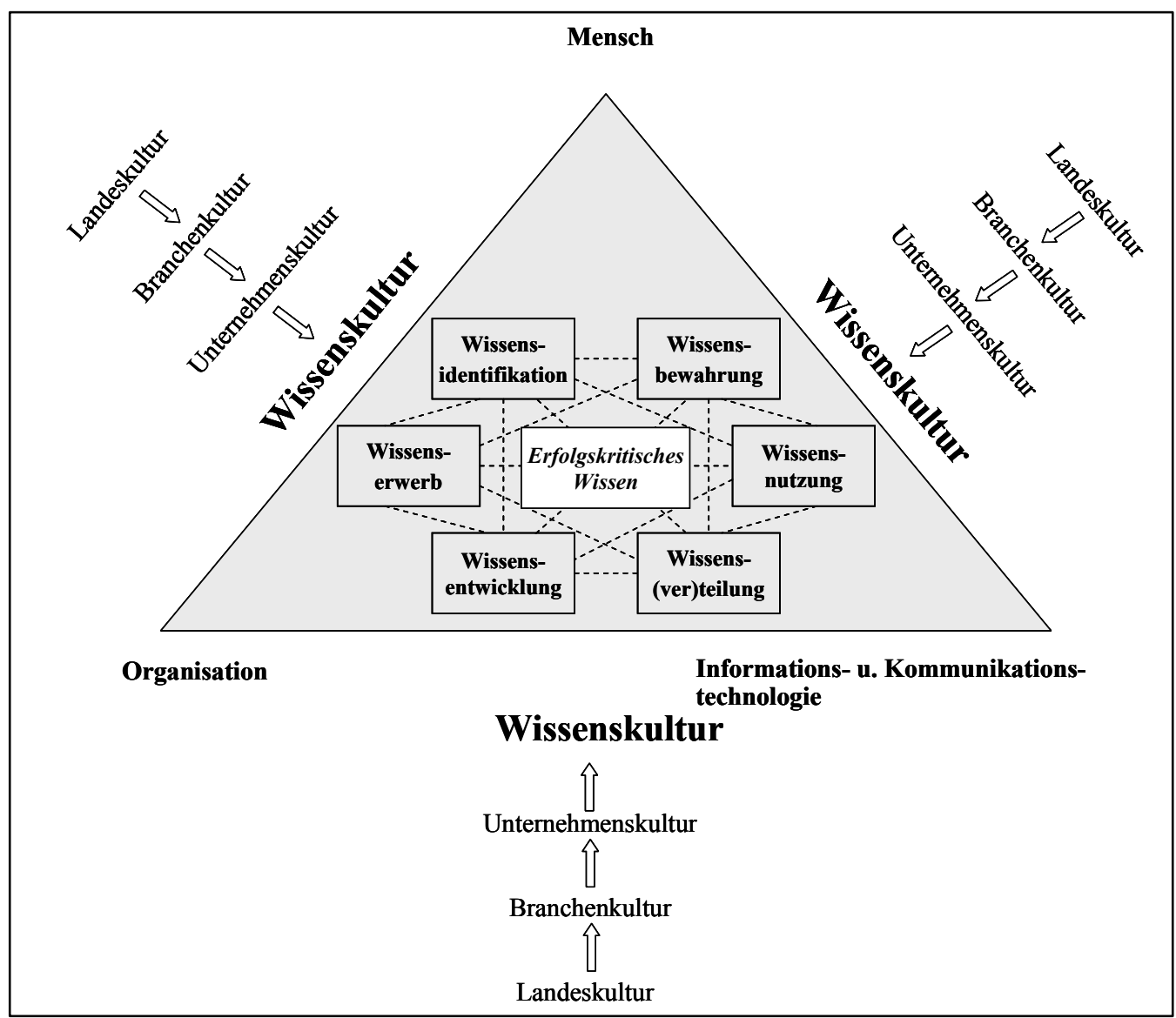

Abb. 1: Gestaltungsfelder eines ganzheitlichen Wissensmanagements

Quelle: $\quad$ Eigene Darstellung in Anlehung an Probst u.a. (2003), S. 28

Die drei Gestaltungsfelder sind in die Wissenskultur eines Unternehmens eingebettet. Diese bildet die Basis für alle wissensrelevanten Belange in einem Unternehmen und demzufolge den kritischen Erfolgsfaktor für Wissensmanagement. Die Unternehmenskultur hilft, die Lücke zwischen Technologie und Informationen sowie deren effektive Nutzung durch die Wissensarbeiter zum Nutzen der Unternehmung zu schließen. ${ }^{21}$ Fehlt eine Unternehmenskultur, die soziale Interaktionen fördert, kann keine wissensorientierte Unternehmung entstehen. ${ }^{22}$ Die Unternehmenskultur (Mikrokultur) ist stets in eine Ethnokultur (Makrokultur) eingebettet. Von Ethnokultur wird dann gesprochen, wenn das Kollektiv, dessen Kultur erforscht wird, ein Volk, eine Nation oder eine andere in sich stimmige und geschlossene große Gesamtheit von Personen darstellt. ${ }^{23}$ Menschen sind aufgrund ihrer Geburt einer Landeskultur zugehörig. Sie werden von den der Kultur zugrunde liegenden Annahmen geprägt und eignen sich dadurch Wertvorstellungen an, die später nur bedingt und mit Schwierigkeiten veränderbar sind. ${ }^{24}$ Landes- und Unternehmens-

21 Vgl. Brelade/Harman (2000), S. 28

22 Vgl. Pemberton/Stonehouse (2002), S. 87; Cameron (2002), S. 22.

23 Vgl. Kiechl (1990), S. 107 ff.

24 So sind westliche Kulturen z.B. deutlich stärker durch ein Wettbewerbsdenken gekennzeichnet als ein Großteil der Kulturen im asiatischen Raum. In westlichen Nationen steht der wirtschaftliche Erfolg in direktem Zusammenhang zum gesellschaftlichen Ansehen. In Südamerika glauben die Menschen hingegen vermehrt, dass sie ihrem Schicksal ausgeliefert sind. Vgl. Pieler (2001), S. 147. 
kultur stellen keine isolierten Kulturebenen dar, sondern überlagern bzw. konkurrenzieren sich gegenseitig; diese Entwicklung erfolgt in einer wechselseitigen Abhängigkeit. Beide Kulturen werden von den in diesen Ebenen befindlichen Individuen als ein mehr oder weniger schlüssiges Gesamtsystem und Weltbild wahrgenommen, das als Ganzes die Verhaltensweisen seiner Mitglieder beeinflusst. ${ }^{25}$

\section{Wissenskultur}

Unternehmenskultur wurde sehr lange als „,soft stuff ${ }^{\text {‘26 }}$ abgetan, zumal dieses Phänomen nur mit Schwierigkeiten zu handhaben, mit viel Mühe zu ändern und beinahe unmöglich zu messen ist. ${ }^{27}$ Seit Beginn der 80er Jahre hat diese Thematik sowohl in der Praxis als auch in der Wissenschaft viel Aufmerksamkeit erhalten. ${ }^{28}$ Die Bedeutung der Unternehmenskultur für den Unternehmenserfolg liegt u.a. im laufenden Wandel, der an Schnelligkeit und Veränderungsdynamik zunimmt. Die vielfältigen Herausforderungen der $\mathrm{Zu}$ kunft sind nicht mehr mit Rezepten der Vergangenheit, wie Lean Management oder Kostentechnokratie zu bewältigen. Es braucht bei den Mitarbeitern einen neuen Geist, der diese Veränderungen begleitet und vorantreibt anstatt sie zu verhindern. Der Wandel verlangt bei hohem Tempo eine kontinuierliche Entwicklung von neuen Betrachtungsweisen über das Funktionieren der Märkte, über die Art und Weise der Arbeitsorganisation, über die gegenseitige Zusammenarbeit und den eigenen Umgang mit Neuerungen. Um mit komplexen Vorgängen, Schnelligkeit, steter Veränderung und neuen Umgangsformen umgehen zu können und nicht von den laufenden technischen Entwicklungen und dem Wandel der kommenden Jahren abgehängt zu werden, sind kulturelle Kompetenz sowie das Beherrschen von Kulturtechniken ${ }^{29}$ unerlässlich. ${ }^{30}$ Heute stimmt man in Wissenschaft und Praxis überein, dass der Unternehmenserfolg in hohem Maße von der Unternehmenskultur abhängt: „Without exception, companies that outperformed their industry peers excelled at what we call the four primary management practices - strategy, execution, culture, and structure". ${ }^{31}$

Dem Begriff Wissenskultur wird in verschiedenen Wissenschaftsdisziplinen, wie z.B. Anthropologie, Soziologie, Geschichte und Betriebswirtschaftslehre eine unterschiedliche Bedeutung zugeschrieben. ${ }^{32}$ In der Betriebswirtschaftslehre wird darunter nicht etwa der Begriff der Hochkultur, also der literarischen und künstlerischen Errungenschaften einer Zivilisation, sondern ein Teil der Unternehmenskultur verstanden. ${ }^{33}$ Die Unterneh-

25 Vgl. Schreier/Thommen (2002), S. $411 \mathrm{f}$.

26 Juechter u.a. (1998), S. 63. So hat Eberhard Dülfer z.B. geschrieben. „Noch um 1980 hätte in der Wirtschaftspraxis jedermann einen scharfen Trennstrich zum kulturellen Bereich gezogen. ,Kultur’ wurde mit Kunst, Spiel und Unterhaltung, mit Freizeit und Emotion assoziiert [...]“ (1991), S. 2.

27 Vgl. Juechter u.a. (1998), S. 64.

28 Vgl. May (1997), S. 31.

29 Beispiele von Kulturtechniken zeigen sich in der Benutzung von Apparaten, technischer Systeme und neuer Medien oder in der Anwendung methodischer Verfahrensweisen. Vgl. Wagner (1999), o. S.

30 Vgl. Schmelcher u.a. (2002), S. 13 f.

31 Nohria u.a. (2003), S. 43.

32 Vgl. z.B. Fried/Kailer (2003); Sandkühler (2002).

33 Vgl. Sackmann (2002), S. 38. 
menskultur und die Wissenskultur sind demnach eng miteinander verknüpft. Es ist davon auszugehen, dass die Wissenskultur stets nur einen oder mehrere Teilaspekte der Unternehmenskultur umfasst, die für ein erfolgreiches Wissensmanagement von zentraler Bedeutung sind. ${ }^{34}$ Zahlreiche Autoren sind sich über den entscheidenden Stellenwert einer Wissenskultur für den Erfolg von Wissensmanagement einig: „Die Förderung einer Knowledge Culture sowie die Erzielung eines kulturellen Wandels ist sicherlich eine der größten Herausforderungen des Wissensmanagements. Häufig wird in ihr der entscheidende Erfolgsfaktor gesehen, der über den Erfolg bzw. Misserfolg aller geplanten Maßnahmen entscheidet" ". ${ }^{35}$

Für die Bestimmung der Wissenskultur werden jene Aspekte der Unternehmenskultur herauskristallisiert, die für die Einführung und Integration eines ganzheitlichen Wissensmanagements eine zentrale Rolle spielen. Vom Grad ihrer Ausprägung hängt es $a b$, ob Prozesse des Wissensmanagements unterstützt, behindert oder sogar verunmöglicht werden. Dabei zeigte sich, dass insbesondere die Werte Vertrauen, Zusammenarbeit, Offenheit, wahrgenommene Autonomie, Lernbereitschaft und Fürsorge eine wissensfreundliche Unternehmenskultur auszeichnen. ${ }^{36}$ Demzufolge wird Wissenskultur wie folgt definiert: „Die Wissenskultur ist Teil der Unternehmenskultur und umfasst die Gesamtheit der Normen und Werte in einer Unternehmung, die die Denk- und Verhaltensweisen der Unternehmensmitglieder im täglichen Umgang mit Wissen prägen. Werte der Wissenskultur sind Vertrauen, Zusammenarbeit, Offenheit, wahrgenommene Autonomie, Lernbereitschaft und Fürsorge“، ${ }^{37}$

\section{Empirische Untersuchungen und Ergebnisse}

Im Rahmen einer Intensivfallstudie wurde in der Schweizerischen Post der Zusammenhang zwischen den beiden Konstrukten „Wissenskultur“ und „Wissensmanagement“ sowie mögliche Einflussfaktoren der Wissenskultur untersucht. Die Untersuchung fokussiert sich auf vier Geschäftsbereiche der Schweizerischen Post, die mit rund 52000 Mitarbeitern die zweitgrößte Arbeitgeberin der Schweiz ist. Die Untersuchungsbereiche ExpressPost, PaketPost, ${ }^{38}$ PostFinance und PostMail haben externen Kundenkontakt, sind jedoch unterschiedlich lange von der außerbetrieblichen Rahmenbedingung „Wettbewerb" betroffen. Die Post ist ein öffentlich-rechtliches Unternehmen, das aufgrund des neuen Postrechts stufenweise mit einem liberalisierten Postmarkt konfrontiert wird. Der Bundesrat (die Schweizer Bundesregierung) als Eigner erwartet von der Post Wettbewerbsfähigkeit, Kundenorientierung und Eigenwirtschaftlichkeit. ${ }^{39}$

34 Vgl. Armutat u.a. (2002), S. 36; Bohinc (2003), S. 376.

35 Alex u.a. (2002), S. 62; Thom (2003), S. 11.

36 Für eine ausführliche Beschreibung der einzelnen Werte vgl. Sollberger (2004), S. 32 ff.

37 Sollberger (2004), S. 39.

38 Als eigenständige Untersuchungseinheiten wurden bei PaketPost die drei Paketzentren untersucht.

39 Vgl. Fischer (2000), S. 222. 
Folgende Hypothesen wurden untersucht:

- Hypothese 1: Bereiche mit einer stärker ausgeprägten Wissenskultur weisen höhere Werte im Umgang mit Wissen auf.

- Hypothese 2: Bereiche innerhalb der Schweizerischen Post, die im Wettbewerb stehen, haben eine stärker ausgeprägte Wissenskultur.

- Hypothese 3: Organisationseinheiten mit einer stärker ausgeprägten Wissenskultur weisen eine höhere Produktivität auf. ${ }^{40}$

Abhängig vom Kulturverständnis eines Forschers wird zur Untersuchung der Unternehmens- bzw. Wissenskultur ein quantitativer resp. qualitativer Ansatz gewählt. ${ }^{41}$ Beide Forschungsansätze weisen sowohl Vor- als auch Nachteile auf (vgl. Tabelle 1).

\begin{tabular}{|c|c|c|c|}
\hline \multicolumn{4}{|c|}{ Qualitative und quantitative Methoden zur Untersuchung der Unternehmenskultur } \\
\hline \multicolumn{2}{|c|}{ Qualitative Untersuchungsmethode } & \multicolumn{2}{|c|}{ Quantitative Untersuchungsmethode } \\
\hline Vorteile & Nachteile & Vorteile & Nachteile \\
\hline $\begin{array}{l}\text { Zugang zur } \\
\text { Einzigartigkeit jeder } \\
\text { Unternehmenskultur } \\
\\
\text { Vgl. Sackmann (1991), } \\
\text { S. } 299 \text { f.; vgl. Smircich } \\
\text { (1983), S. 354; } \\
\text { vgl. Neubauer (2003), } \\
\text { S. } 22\end{array}$ & $\begin{array}{l}\text { Oftmals fehlende } \\
\text { Repräsentativität, da } \\
\text { Untersuchung von } \\
\text { Spezialfällen } \\
\text { Vgl. Weber/Camerer } \\
\text { (2003), S. } 403 \text { ff.; } \\
\text { vgl. Sackmann (1991), } \\
\text { S. } 299\end{array}$ & $\begin{array}{l}\text { Standardisierung der } \\
\text { Ergebnisse und Umgang } \\
\text { mit großen Datenmengen } \\
\text { gegeben } \\
\text { Vgl. Sackmann (1991), } \\
\text { S. } 299 \text {; vgl. De } \\
\text { Witte/Muijen } \\
\text { (1999), S. 590; } \\
\text { vgl. Neubauer (2003), } \\
\text { S. } 21 \text { f. }\end{array}$ & $\begin{array}{l}\text { Vorkenntnisse zur } \\
\text { Entwicklung geeigneter } \\
\text { Instrumente notwendig } \\
\text { Vgl. De Witte/Muijen (1999), } \\
\text { S. } 590 ; \\
\text { vgl. Unterreitmeier/ } \\
\text { Schwinghammer (2004), S. } 9\end{array}$ \\
\hline $\begin{array}{l}\text { Detailliertere } \\
\text { Informationen, somit } \\
\text { klareres Bild des } \\
\text { untersuchten } \\
\text { Unternehmens } \\
\text { Vgl. Geertz (1993), } \\
\text { S. } 5 \text { ff.; vgl. Schein } \\
\text { (1992), S. } 147 \text { ff. }\end{array}$ & $\begin{array}{l}\text { Eingeschränkte } \\
\text { Vergleichbarkeit mit } \\
\text { anderen Unternehmen } \\
\text { bzw. } \\
\text { Untersuchungen } \\
\text { Vgl. Delobbe et al. } \\
\text { (2002), S. } 3\end{array}$ & $\begin{array}{l}\text { Messbarkeit kultureller } \\
\text { Veränderungen über die } \\
\text { Zeit } \\
\text { Vgl. Hofstede et al. (1990), } \\
\text { S. } 313\end{array}$ & $\begin{array}{l}\text { Beschränkung auf } \\
\text { beobachtbare und messbare } \\
\text { Manifestationen } \\
\text { Vgl. Ashkanasy et al. (2000), } \\
\text { S. 132; vgl. Kolbeck/Nicolai } \\
\text { (1996), S. } 193\end{array}$ \\
\hline $\begin{array}{l}\text { Erhaltung der } \\
\text { Individualität der } \\
\text { einzelnen Probanden } \\
\text { Vgl. Jauch et al. } \\
\text { (1980), S. } 518\end{array}$ & $\begin{array}{l}\text { Gefahr einer starken } \\
\text { Beeinflussung durch } \\
\text { den Forscher } \\
\text { Vgl. De Witte/Muijen } \\
\text { (1999), S. } 589\end{array}$ & $\begin{array}{l}\text { Vergleichbarkeit mit } \\
\text { anderen } \\
\text { Forschungsergebnissen } \\
\text { bzw. Unternehmen } \\
\text { möglich } \\
\text { Vgl. Denison/Mishra } \\
\text { (1995), S. 206 }\end{array}$ & $\begin{array}{l}\text { Geringer Einbezug von } \\
\text { Kontextinformationen } \\
\text { Vgl. De Witte/Muijen } \\
\text { (1999), S. } 590\end{array}$ \\
\hline
\end{tabular}

Tab. 1: Qualitative und quantitative Forschungsmethoden für Kulturassessments

Quelle: $\quad$ Sollberger (2005), S. 14

Aus diesem Grund wird vermehrt die Ansicht vertreten, dass für die Erfassung des komplexen Konstrukts „Unternehmenskultur“ die Kombination verschiedener Untersuchungsverfahren notwendig ist und sich ein Methodenpluralismus als zielführend erweist. $^{42}$ Die Forschungsstrategie der vorliegenden Intensivfallstudie besteht aus einer

40 Vgl. Sollberger (2005), S. 4.

41 Vgl. Smircich (1983), S. 339 ff.

42 Vgl. Davies/Fitchett (2004), S. 318 
quantitativen sowie einer qualitativen Vorgehensweise und verfolgt demnach die beschriebene Methodentriangulation.

1. Empirische Untersuchungen

a) Quantitative Untersuchung

Im Rahmen einer quantitativen Untersuchung wurden Primärdaten aus einer jährlich wiederkehrenden Gesamterhebung zu den Themen Personalzufriedenheit und Kultur im Hinblick auf die aufgestellten Hypothesen retrospektiv ausgewertet. Der verwendete Fragebogen beruht auf dem Leistungskultur-Modell von Daniel R. Denison. ${ }^{43}$ Aus den ca. 100 Items wurden mit Hilfe von Literaturanalyse und Expertengesprächen jene Fragen herauskristallisiert, die Aufschluss über die vorhandene Wissenskultur sowie über die Kernprozesse des Wissensmanagements ${ }^{44}$ geben. In der vorliegenden Untersuchung geht es um die Überprüfung einer als kausal angenommenen Abhängigkeit zwischen den theoretischen Konstrukten „Wissenskultur“ und „Wissensmanagement“. Theoretische Konstrukte lassen sich nicht direkt messen und werden aus diesem Grund oft auch als latente Variablen bezeichnet. ${ }^{45}$ Latente Variablen werden mit Hilfe von manifesten Variablen bzw. Indikatoren indirekt gemessen. ${ }^{46}$ Gemäß dem Partial-Least-Squares-Ansatz (PLS) wurde der angenommene positive Zusammenhang zwischen den beiden latenten Konstrukten „Wissenskultur“ und „Wissensmanagement“ überprüft. Der PLS-Ansatz ist die Kombination einer Hauptkomponentenanalyse und einer multiplen Regression. ${ }^{47}$ Aus den Indikatoren werden durch die Hauptkomponentenanalyse Themenbereiche bzw. latente Variablen bestimmt, deren Einfluss auf die abhängige Variable mittels Regression untersucht wird. Es handelt sich um ein iteratives Verfahren, in dem abwechslungsweise Hauptkomponentenanalyse und Regression gerechnet werden, bis der Algorithmus konvergiert. Abschließend ermittelt der PLS-Ansatz gewichtete Mittelwerte für latente und manifeste Variablen sowie Ortungsparameter. ${ }^{48}$

\section{b) Qualitative Untersuchung}

Nachgelagert wurden die Ergebnisse der quantitativen Studie anhand der qualitativen Methode „ermittelnde Gruppendiskussion“ analysiert. Diese Methode hat die „Erlangung

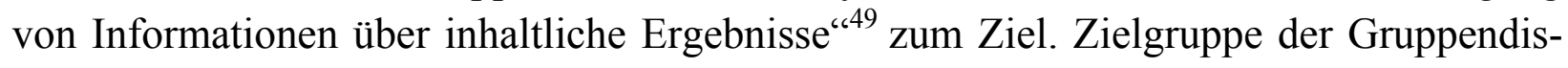
kussionen ist das mittlere Management, da dieses eine Brückenfunktion zwischen dem

43 Vgl. Denison (1997).

44 Vgl. Probst u.a. (2003), S. 28 ff.

45 Vgl. Homburg/Giering (1996), S. 6.

46 Vgl. Chin (1998), S. 299.

47 Vgl. Götz/Liehr-Gobbers (2004), S. 722 ff.

48 Vgl. Herrmann u.a. (2004), S. 6; Götz/Liehr-Gobbers (2004), S. 722 ff.

49 Lamnek (1995), S. 134. 
Topmanagement und den Mitarbeitern der Basis besitzt und dadurch die Wissenskultur in hohem Maße beeinflusst. ${ }^{50}$

Mit der Durchführung von Gruppendiskussionen soll einerseits eine vertiefte Perspektive der Wissenskultur gewonnen werden, andererseits sind die Ergebnisse aus der quantitativen Untersuchung zu interpretieren und zu plausibilisieren. Der entwickelte Leitfaden für die sieben Gruppendiskussionen mit acht bis zehn Teilnehmern basiert auf den gezeigten theoretischen Grundlagen und auf den Ergebnissen der quantitativen Auswertung. Die Interpretation der zusammenfassenden Protokolle erfolgte anhand der qualitativen Inhaltsanalyse nach Mayring. ${ }^{51}$

\section{Ergebnisse}

\section{a) Quantitative Ergebnisse}

- Hypothese 1: In der vorliegenden Untersuchung wurde für die aggregierten Stichproben zwischen der unabhängigen Variable Wissenskultur (gewichteter Mittelwert $=62.31$ ) und der abhängigen Variable Wissensmanagement (gewichteter Mittelwert $=66.81$ ) ein $\beta$ von 0.90 und ein Konfidenzintervall von 0.158 ermittelt, was einem sehr starken Einfluss entspricht. Damit wird Hypothese 1 erhärtet und das entwickelte Strukturgleichungsmodell aufgrund des hohen $\mathrm{R}^{2}\left(\mathrm{R}^{2}=0.81\right)$ und $\beta$ Koeffizienten sowie der hohen Alpha-Werte als stabil betrachtet. ${ }^{52}$

- Hypothese 2: Weiter zeigt sich, dass jene Untersuchungsbereiche, die von Anfang an im liberalisierten Markt oder seit längerer Zeit im Wettbewerb tätig sind, eine signifikant höhere Wissenskultur aufweisen als jene Bereiche, die noch mehrheitlich im Monopol agieren. Für die vier Stichproben ExpressPost, PaketPost, PostFinance und PostMail wurde die Wissenskultur als gewichteter Mittelwert im Jahr 2004 berechnet, zwischen den einzelnen Stichproben paarweise verglichen und auf Signifikanz geprüft. Dabei zeigte sich, dass PostFinance eine signifikant höhere Wissenskultur $(\alpha=0.05 ; \mathrm{t}=2.45)$ aufweist als ExpressPost. Diese wiederum hat eine signifikant höhere Wissenskultur $(\alpha=0.01 ; \mathrm{t}=3.25)$ als PaketPost. Zwischen der Wissenskultur von PaketPost und PostMail ist auf dem 5 Prozent-Niveau kein signifikanter Unterschied feststellbar. ${ }^{53}$ Damit lässt sich Hypothese 2 erhärten.

- Hypothese 3: Hypothese 3 wurde während eines Untersuchungszeitraumes von drei Jahren in den drei Paketzentren der Post überprüft, die gleichzeitig gebaut und Mitte 1999 in Betrieb genommen wurden. Hinsichtlich ihrer technischen Ausstattung und Rahmenbedingungen sind sie nahezu identisch.

Befunde zur Hyphthese 3 sind:

50 Vgl. Milner (2000), S. 73 f.

51 Vgl. Mayring (2003).

52 Vgl. Gefen u.a. (2000), S. 36 f.

53 Die Wissenskultur von PaketPost ist auf dem 15-Prozent-Signifikanzniveau $(t=0,87)$ höher als diejenige von PostMail. 
Für 2004 zeigt sich, dass Paketzentrum 1 mit der höchsten Produktivität $t^{54}(131.13)^{55}$ gegenüber den beiden anderen Zentren auch die höchste Wissenskultur (62.3) aufweist. Paketzentrum 2 zeigt die zweithöchste Produktivität (126.82) und die zweithöchste Wissenskultur (59.52). Jedoch ist zwischen der Wissenskultur von Paketzentrum 2 und derjenigen von Paketzentrum 3 kein signifikanter Unterschied auf dem 5-Prozent-Niveau feststellbar. ${ }^{56}$ Für 2004 kann die Hypothese nicht vollumfänglich erhärtet werden.

Im Jahr 2003 hat Paketzentrum 1 wiederum die signifikant höchste Wissenskultur (66.59) und die höchste Produktivität (123.05). Paketzentrum 2 (116.88) und Paketzentrum 3 (117.56) besitzen eine vergleichbare Produktivität. Zwischen der Wissenskultur der beiden Zentren besteht keine signifikante Differenz. ${ }^{57}$ Aufgrund dieser Ergebnisse lässt sich die Hypothese für 2003 erhärten.

Im Jahr 2002 weisen Paketzentrum 1 (104.64) und Paketzentrum 3 (104.87) eine nahezu identische Produktivität auf. Der Vergleich ihrer Wissenskultur zeigt keinen signifikanten Unterschied. Paketzentrum 2 mit einer deutlich tieferen Produktivität (98.97) hat gegenüber Paketzentrum $1(\alpha=0.05 ; \mathrm{t}=3.39)$ und Paketzentrum $3(\alpha=0.01 ; \mathrm{t}=4.24)$ auch eine signifikant tiefere Wissenskultur. Für dieses Untersuchungsjahr lässt sich die Hypothese erhärten.

Die Ergebnisse der Hypothesenüberprüfung wurden in mehreren Gesprächen mit den Leitern der Paketzentren diskutiert, um den situativen Kontext in die Interpretation mit einzubeziehen. ${ }^{58}$ Über alle drei Untersuchungsjahre lässt sich die Hypothese, dass eine hohe Wissenskultur zu einer hohen Produktivität führt, nicht vollständig untermauern. Jedoch konnte für die gesamte Periode gezeigt werden, dass das Paketzentrum mit der höchsten Wissenskultur stets auch die höchste Produktivität aufweist. Diese Ergebnisse sowie die verschiedenen Gespräche mit den Paketzentrenleitern weisen darauf hin, dass ein positiver Zusammenhang zwischen den beiden Größen als existent zu betrachten ist.

\section{b) Qualitative Ergebnisse}

Bei der Beurteilung der Wissenskultur zeigt sich, dass in allen Untersuchungsbereichen zwar sehr viel dokumentiert, die Verwendbarkeit der Dokumente jedoch bezweifelt wird, da diese teils nicht mehr gefunden, teils nicht laufend aktualisiert werden. Weiter weisen die Interviews auf die Bedeutung informeller Netzwerke und persönlicher Kontakte für

54 Die Produktivität wird als Anzahl verarbeitete Pakete pro Mitarbeiter und Stunde gemessen.

55 Die Differenz eines Indexpunktes in der Produktivität schlägt sich unter finanziellem Gesichtspunkt als Durchschnitt über alle drei Zentren und pro Jahr mit CHF 150000 nieder.

56 Der Unterschied in der Wissenskultur zwischen Paketzentrum 2 und Paketzentrum 3 ist auf dem 20-ProzentNiveau $(\mathrm{t}=2.46)$ signifikant.

57 Die Differenz in der Wissenskultur zwischen Paketzentrum 2 und Paketzentrum 3 ist auf dem 15-ProzentNiveau $(\mathrm{t}=3.26)$ signifikant.

58 Vgl. das Gespräch mit dem Paketzentrumsleiter von Härkingen und dem Leiter aller drei Zentren vom 7. Dezember 2004; vgl. das Gespräch mit den Leitern der drei Paketzentren und dem Leiter aller drei Zentren vom 13. Januar 2005. 
einen erfolgreichen Wissenstransfer hin. Der Einfluss der abgefragten Faktoren ${ }^{59}$ auf die Wissenskultur wird in allen Bereichen vergleichsweise ähnlich beurteilt. So wird insbesondere dem externen Wettbewerb, dem Management, der Aus- und Weiterbildung sowie der organisatorischen Rahmenstruktur ein positiver, der Mehrsprachigkeit ${ }^{60}$ und dem ausgeprägten internen Wettbewerb hingegen ein negativer Einfluss auf die Wissenskultur beigemessen. Zudem zeigte sich, dass die aufgrund persönlicher Einschätzung vorgenommene Reihenfolge der Subwissenskulturen in den Untersuchungsbereichen mit den Ergebnissen der quantitativen Studie übereinstimmt. Gründe für die unterschiedlich ausgeprägten Wissenskulturen werden primär im externen Wettbewerb, der Bereichsgröße sowie in der Professionalisierung der Mitarbeiter gesehen.

\section{Handlungsempfehlungen}

In der Praxis hat sich gezeigt, dass die Entwicklung einer Wissenskultur auf mehreren organisatorischen Ebenen und über längere Zeit stattfinden muss. ${ }^{61}$ Einerseits gilt es, gemäß der vorherrschenden Kultur und den Rahmenbedingungen einer Unternehmung, wie Branche und Mitarbeiterzahl geeignete Instrumente des Wissensmanagements zu etablieren. Werden die Instrumente des Wissensmanagements regelmäßig angewendet und erfährt der einzelne Mitarbeiter einen Nutzen, kann sich auf diese Weise allmählich eine Wissenskultur entwickeln. Diese Ebene setzt bei den drei Gestaltungsfeldern des Wissensmanagements (Mensch, Organisation sowie Informations- und Kommunikationstechnologie) an. Andererseits sollen parallel zu den Verbesserungen bei den drei Gestaltungsfeldern mit Hilfe des Change-Managements ${ }^{62}$ die Werte einer Wissenskultur gefördert und allmählich eine wissensfreundliche Unternehmenskultur entwickelt werden. ${ }^{63}$ Für diese Ebene sind die Führungskräfte ausschlaggebend. Die gewünschten Werte sind vorzuleben, die notwendigen Veränderungsmaßnahmen einzuleiten und Ressourcen freizugeben. Zweifelsohne nimmt die Führung damit auch Einfluss auf die Gestaltungsfelder eines ganzheitlichen Wissensmanagements.

Nachfolgend werden exemplarisch für die drei Gestaltungsfelder Mensch, Organisation sowie Informations- und Kommunikationstechnologie eines ganzheitlichen Wissensma-

59 In den Gruppendiskussionen wurden folgende Einflussfaktoren der Wissenskultur diskutiert: externer Wettbewerb, Mehrsprachigkeit, Jargon/Fachsprache, Management/Vorgesetzte, Organisation, Information und Kommunikation, Aus- und Weiterbildung, Anreizsysteme, Informationstechnologie, Initiativen „Wissensmanagement des Konzerns".

60 Die Schweizerische Post besitzt mit Deutsch, Französisch und Italienisch drei offizielle Unternehmenssprachen.

61 Gemäß Sackmann haben Maßnahmen zur Kulturveränderung und -gestaltung beim Mitarbeiter als Kulturträger, den Kontextbedingungen und den Führungskräften anzusetzen. Vgl. Sackmann (2002), S. 171.

62 Das Konzept des Change Managements umfasst alle geplanten, gesteuerten, organisierten und kontrollierten Veränderungen in den Strategien, Prozessen, Strukturen und in den Kulturen sozio-ökonomischer Systeme. Vgl. Thom (1997b), S. $201 \mathrm{f}$.

63 Vgl. Kyriakidou (2004), S. 22 f.; vgl. Grüter (2004), S. 10. 
nagements und für die Führungsebene mögliche Gestaltungsmaßnahmen zur Förderung und längerfristigen Entwicklung einer Wissenskultur erläutert. ${ }^{64}$

1. Implikationen für die Gestaltungsfelder eines ganzheitlichen Wissensmanagements

\section{a) Mensch}

Der Mensch ist der zentrale Wissensträger in einer Unternehmung. Von seiner Leistungsbereitschaft und -fähigkeit hängt es letztlich ab, inwiefern der Austausch und die Entwicklung von Wissen erfolgen. ${ }^{65}$ Verschiedene Faktoren können die Leistungsbereitschaft und -fähigkeit beeinflussen. Entscheidend für den Unternehmenserfolg ist nicht das Wissen des einzelnen Mitarbeiters, sondern die Kombination des individuellen Wissens durch Vernetzung der individuellen Wissensträger. ${ }^{66}$ Wissen wird jedoch nur geteilt und angewendet, wenn sich die Mitarbeiter ausreichend und in erster Linie persönlich kennen und sich gegenseitig vertrauen. In diesem Kontext wurde in allen Gruppendiskussionen wiederholt auf die große Bedeutung von informellen Netzwerken wie Communities of Practice hingewiesen. Communities of Practice sind informelle Gruppen von Mitarbeitern, die sich selber organisieren und auf freiwilliger und persönlicher Basis treffen, um zu einem bestimmten Arbeitsgebiet Erfahrungen und Wissen auszutauschen und neue Lösungen zu entwickeln. Die Mitglieder können aus verschiedenen Bereichen und Hierarchiestufen stammen. Die Beteiligung erfolgt für jene Zeit, in der ein persönlicher Nutzen ersichtlich ist. Gerade für den Wissenstransfer spielt diese Arbeitsform eine entscheidende Rolle. ${ }^{67}$ Mit Hilfe von Communities of Practice sollen neben neuen Netzwerken insbesondere auch bereits bestehende, jedoch versteckte Netzwerke gefördert und offen gelegt werden. Positiv an den Communities ist u.a. die freiwillige Teilnahme. Dadurch kann eher davon ausgegangen werden, dass die Bereitschaft für einen Wissenstransfer vorhanden ist. Durch persönliche Treffen lässt sich eine Vertrauensbasis schaffen, die den Wissensaustausch fördert. Gerade für neue Mitarbeiter sind diese Netzwerke sehr hilfreich, da benötigte Kontakte sehr schnell herzustellen sind und nicht erst über längere Zeit aufgebaut werden müssen. Auch für die Wissensbewahrung spielen Communities of Practice eine wichtige Rolle, wird doch das Wissen einzelner Spezialisten einer Vielzahl von Mitarbeitern weitergegeben.

64 Die Gestaltungsempfehlungen basieren auf der Intensivfallstudie in der Schweizerischen Post und können demzufolge nicht unbesehen in anderen Branchen und Unternehmen umgesetzt werden. Für den vorliegenden Artikel wurden jedoch bewusst Implikationen ausgewählt, die sich durch eine hohe Übertragbarkeit auszeichnen.

65 Vgl. Grüter (2004), S. 10; Thom (2002), S. 181; Thom/Harasymowicz-Birnbach (2003b), S. 52.

66 Vgl. Felbert (1998), S. 139.

67 Vgl. Davenport/Probst (2002), S. 16. 


\section{b) Organisation}

Zwingende Voraussetzung für den Wissensaustausch ist das Vorhandensein einer gemeinsamen Landes- und Fachsprache. Insbesondere in einer mehrsprachigen oder internationalen Unternehmung mit zahlreichen Fachbereichen ist diese Rahmenbedingung eine große Herausforderung. Für neu eingetretene Mitarbeiter stellen der Unternehmensjargon und dessen Abkürzungen eine nicht zu unterschätzende Schwierigkeit dar. Zentrale Dokumente wie Protokolle auf Stufe Geschäftsleitung oder Projektberichte sollten daher stets ein Abkürzungsverzeichnis und eine Erklärung selten verwendeter Fachbegriffe enthalten.

\section{c) Informations- und Kommunikationstechnologie}

Die befragten Mitarbeiter weisen dem Intranet als Informationsspeicher eine wichtige Bedeutung zu. Allerdings zeigen sich beim Ausschöpfen des vorhandenen Potenzials zur Informationsgewinnung erhebliche Schwierigkeiten, da es teilweise an AnwendungsKnow-how fehlt. Für neue Mitarbeiter oder auch Mitarbeiter, die aufgrund ihrer Funktion nicht regelmäßig mit dem Intranet arbeiten, ist dieses Medium oft ein Buch mit sieben Siegeln. Fehlende Kenntnisse über die im Intranet vorhandenen Inhalte und deren Auffindbarkeit verunmöglichen eine erfolgreiche Nutzung. Um dieses Problem zu beheben, sind kurze Einführungen ins Intranet auf freiwilliger Basis anzubieten. Einerseits gilt es hier, die Gesamtstruktur des Intranets und jene Inhalte zu erläutern, die von allen Organisationseinheiten regelmäßig verwendet werden. Andererseits ist auf den spezifischen Intranetauftritt großer Bereiche einzugehen.

\section{Implikationen für die Führung}

Die Ergebnisse der quantitativen Untersuchung belegen den positiven Zusammenhang zwischen Wissenskultur und Wissensmanagement sowie mehrheitlich zwischen Wissenskultur und Produktivität. Für ein zielorientiertes Wissensmanagement und eine erfolgreiche Unternehmung gilt es daher, die Werte der Wissenskultur zu fördern und $\mathrm{zu}$ entwickeln. In diesem Zusammenhang spielt das Führungsverhalten eine entscheidende Rolle. ${ }^{68}$ Die große Wirkung des Managements als Vorbild sowie in der Unterstützung und Förderung des Wissenstransfers wurde auch in den Gruppendiskussionen betont. Besondere Bedeutung kommt der operativen Ebene bzw. der täglichen Interaktion zwischen Management und Mitarbeitern zu. Aus diesem Grund ist es zentral, dass die Führungspersonen regelmäßig im Betrieb sind. Die Mitarbeiter sehen auf diese Weise, dass ihre Probleme ernst genommen und ihre Arbeit geschätzt wird. Die Führung hingegen wird direkt mit den Fragestellungen des operativen Bereichs konfrontiert. In den Gruppendiskussionen wurde die Absolvierung von Praktika durch Führungskräfte im Betrieb als sehr

68 Vgl. Thom (1997a), S. 12. 
positiv empfunden. Es ist sicherzustellen, dass die Mitglieder der ersten und zweiten Führungsebene jährlich mindestens einen mehrtägigen Arbeitseinsatz im Betrieb leisten und zusätzlich regelmäßig den Betrieb aufsuchen. So haben Mitarbeiter im direkten Dialog die Möglichkeit, Fragen zu stellen oder auf Schwierigkeiten hinzuweisen, wodurch sich allmählich eine Kultur des Vertrauens und der Offenheit entwickeln kann. Generell hat die direkte Kommunikation zwischen dem Management und den Mitarbeitern eine hohe Effizienz und ist der unpersönlichen Information vorzuziehen.

\section{Schlusswort}

Die Ergebnisse des Forschungsprojekts veranschaulichen die positive Wirkung einer wissensfreundlichen Unternehmenskultur beim Umgang mit Wissen und für die Produktivität einer Unternehmung. Weiter konnte gezeigt werden, dass z.B. die Einflussfaktoren externer Wettbewerb sowie Aus- und Weiterbildung einen ausgesprochen positiven, die Mehrsprachigkeit, die zahlreichen Abkürzungen sowie eine steigende Mitarbeiterzahl pro Bereich einen eher negativen Einfluss auf die Wissenskultur haben. Um mittel- und längerfristig eine Wissenskultur zu fördern und zu entwickeln, kommt dem Management in zweierlei Hinsicht eine entscheidende Rolle zu. Einerseits fungieren die Manager aufgrund ihrer Führungsfunktion als Repräsentanten und Multiplikatoren der vorhandenen Unternehmens- bzw. Wissenskultur, andererseits prägen sie die Gestaltungsfelder Mensch, Organisation sowie Informations- und Kommunikationstechnologie eines ganzheitlichen Wissensmanagements.

\section{Literaturverzeichnis}

Adelsberger, Heimo H., Markus Bick und Thomas Hanke (2002), Einführung und Etablierung einer Kultur des Wissensteilens in Organisationen, in: Virtuelle Organisationen und neue Medien, hrsg. von Martin Engelien und Jens Homann, Köln, S. 529-552

Alex, Björn, Dieter Becker und Jan Stratmann (2002), Ganzheitliches Wissensmanagement und wertorientierte Unternehmensführung, in: Wissensmanagement. Zwischen Wissen und Nichtwissen, hrsg. von Georg Götz, 4. Aufl., Stuttgart, S. 47-69

Armutat, Sascha u.a. (2002), Wissensmanagement erfolgreich einführen. Strategien - Instrumente - Praxisbeispiele, hrsg. von der Deutschen Gesellschaft für Personalführung e.V., Düsseldorf

Ashkanasy, Neal M., Lyndelle E. Broadfoot und Sarah Falkus (2000), Questionnaire Measures of Organizational Culture, in: Handbook of Organizational Culture \& Climate, hrsg. von Neal M. Ashkanasy u.a., Thousand Oaks u. a., S. 131-145

Augustin, Siegfried (2000), Der Stellenwert des Wissensmanagements im Unternehmen, in: Wissensmanagement, hrsg. von Heinz Mandl und Gabi Reinmann-Rothmeier, München u. a., S. 159-168

Birkinshaw, Julian (2001), Why is Knowledge Management so difficult?, in: Business Strategy Review, 12. Jg. Nr. 1, S. 11-18

Boersma, Arno und Vikash Banwarie, (2004), Winner takes all in Knowledge Battle for Postal Innovation. Treat knowledge as a production factor and let your postal innovation strategy thrive. URL: http://www.postalsolutions.com/download\%20docs/Postal_knowledgemanagement.pdf, 24. Juni 2005

Bohinc, Thomas (2003), Wissenskultur - Begriff und Bedeutung, in: WM 2003: Professionelles Wissensmanagement - Erfahrungen und Visionen, hrsg. von Ulrich Reimer u.a., Bonn, S. 371-379

Brelade, Sue und Chris Harman (2000), Using human resources to put knowledge to work, in: Knowledge Management Review, 3. Jg., Nr. 1, S. 26-29

Cameron, Preston D. (2002), The cure for an ailing structure, in: CMA Management, 76. Jg., Nr. 3, S. 21-23 
Chin, Wynne W. (1998), The Partial Least Squares Approach to Structural Equation Modeling, in: Modern Methods for Business Research, hrsg. von George A. Marcoulides, London, S. 295-336

Cong, Xiaoming und Pandya V. Kaushik (2003), Issues of Knowledge Management in the Public Sector, in: Electronic Journal of Knowledge Management, 1. Jg., Nr. 2, S. 25-33

Davenport, Thomas H. und Gilbert Probst (2002), Knowledge Management Case Book. Siemens Best Practices, 2. Aufl., Berlin und München

Davies, Andrea und James Al Fitchett (2004), „Crossing Culture”: A multi-method enquiry into consumer behaviour and the experience of cultural transition, in: Journal of Consumer Behaviour, 3. Jg., Nr. 4, S. 315-330

Delobbe, Nathalie, Robert R. Haccoun und Christian Vandenberghe (2002), Measuring Core Dimensions of Organizational Culture: A Review of Research and Development of a New Instrument, Arbeitspapier 53, Université catholique de Louvain, Institut d'Administration et de Gestion URL: http://www.iag.ucl.ac.be/recherche/Papers/wp53.pdf, 15. August 2005

Denison, Daniel R. (1997), Corporate Culture and Organizational Effectiveness, Ann Arbor

Denison, Daniel R. und Aneil K. Mishra (1995), Towards a Theory of Organizational Culture and Effectiveness, in: Organizational Science, 6. Jg., Nr. 2, S. 204-223

De Witte, Karel und Jaap J. van Muijen (1999), Organizational Culture: Critical Questions for Researchers and Practitioners, in: European Journal of Work and Organizational Psychology, 8. Jg., Nr. 4, S. 583-595

Dülfer, Eberhard (1991), Organisationskultur: Phänomen - Philosophie - Technologie, in: Organisationskultur, hrsg. von Eberhard Dülfer, 2. erw. Aufl., Stuttgart, S. 1-20

Felbert, Dirk v. (1998), Wissensmanagement in der unternehmerischen Praxis, in: Wissensmanagement. Erfahrungen und Perspektiven, hrsg. von Peter Pawlowsky, Wiesbaden, S. 119-141

Fischer, Gerhard W. (2000), Die gelbe Welt in raschem Wandel. Vom Postdepartement der PTT zum öffentlichen Unternehmen Post, in: Vom Service Public zum Service au Public. Regierung und Verwaltung auf dem Weg in die Zukunft, hrsg. von Raoul Blindenbacher u. a., Zürich, S. 220-226

Fried, Johannes und Thomas Kailer (2003), Wissenskulturen, Berlin

Friedli, Beat (2003), Die Schweizerische Post auf dem Weg zu einem erfolgreichen Wissensmanagement, in: Wissensmanagement im privaten und öffentlichen Sektor. Was können die beiden Sektoren voneinander lernen? hrsg. von Norbert Thom und Joanna Harasymowicz-Birnbach, Zürich, S. 143-150

Gamble, Paul R. und John Blackwell (2001), Knowledge Management. A State of the Art Guide, London/Milford

Geertz, Clifford (1993), The Interpretation of Cultures. Selected Essays, London

Gefen, David, Detmar W. Straub und Marie-Claude Boudreau (2000), Structural Equation Modeling and Regression: Guidelines for Research Practice, in: Communications of the Association for Information Systems, 4. Jg., Nr. 7, S. 1-77

Götz, Olivier und Kerstin Liehr-Gobbers (2004), Analyse von Strukturgleichungsmodellen mit Hilfe der PartialLeast-Squares (PLS)-Methode, in: DBW, 64. Jg., Nr. 6, S. 714-738

Grüter, Josef (2004), Wissen, wie Wissen zu managen ist. Gastkommentar, in: Basler Zeitung, 162. Jg., Nr. 157, 8. März 2004, S. 10

Hansen, Morten T., Nitin Nohria, Nitin und Thomas Tierney (1999), What's your Strategy for Managing Knowledge?, in: Harvard Business Review, 77. Jg., Nr. 2, S. 106-116

Herrmann, Andreas, Frank Huber und Frank Kressmann (2004), Partial Least Squares - Ein Leitfaden zur Spezifikation, Schätzung und Beurteilung varianzbasierter Strukturgleichungsmodelle. Arbeitspapier, Lehrstuhl für BWL und Marketing I, Universität Mainz, Zeitschrift für betriebswirtschaftliche Forschung, S. 1-35

Hofstede, Geert u.a. (1990), Measuring Organizational Cultures: A Qualitative and Quantitative Study across Twenty Cases, in: Administrative Science Quarterly, 35. Jg., Nr. 2, S. 286-316

Homburg, Christian und Annette Giering (1996), Konzeptualisierung und Operationalisierung komplexer Konstrukte. Ein Leitfaden für die Marketingforschung, in: MARKETING ZFP, 18. Jg., Nr. 1, S. 5-24

Hopfenbeck, Waldemar, Manuela Müller und Thomas Peisl (2001), Wissensbasiertes Management. Ansätze und Strategien zur Unternehmensführung in der Internet-Ökonomie, Landberg/Lech

Jauch, Laurence R., Richard N. Osborn und Thornan N. Martin (1980), Structured Content Analysis of Cases: A Complementary Method for Organizational Research, in: Academy of Management Review, 5. Jg., Nr. 4., S. $517-525$

Juechter, Matthew W., Caroline Fischer und Randell J. Alford (1998), Five Conditions for High Performance Cultures, in: Training \& Development, 52. Jg., Nr. 5, S. 63-67

Kiechl, Rolf (1990), Ethnokultur und Unternehmenskultur, in: Die Unternehmenskultur. Ihre Grundlagen und ihre Bedeutung für die Führung der Unternehmung, hrsg. von Charles Lattmann, Heidelberg, S. 107-130

Kolbeck, Christoph und Alexander Nicolai (1996), Von der Organisation der Kultur zur Kultur der Organisation, Marburg

Kyriakidou, Olivia (2004), Developing a Knowledge Sharing Culture, in: Management Services, 48. Jg., Nr. 6, S. $22-23$

Lamnek, Siegfried (1995), Methodologie, München und Weinheim

Lee, Heeseok und Byounggu Choi (2003), Knowledge Management Enablers, Processes, and Organizational Performance: An Integrative View and Empirical Examination, in: Journal of Management Information System, 20. Jg., Nr. 1, S. 179-228 
Lengnick-Hall, Mark L. und Cynthia A. Lengnick-Hall (2003), Human Resource Management in the Knowledge Economy, San Francisco

Lenk, Klaus und Peter Wengelowski (2002), Wissensmanagement für das Verwaltungshandeln, in: Wissenssteuerung und Wissensmanagement in Politik, Wirtschaft und Verwaltung, hrsg. von Thomas Edeling u.a., Opladen, S. $147-165$

Mandl, Heinz und Gabi Reinmann-Rothmeier (2000), Die Rolle des Wissensmanagements für die Zukunft. Von der Informations- zur Wissensgesellschaft, in: Wissensmanagement, hrsg. von Heinz Mandl und Gabi ReinmannRothmeier, München u.a., S. 1-17

May, Stefan (1997), Organisationskultur. Zur Rekonstruktion und Evaluation heterogener Ansätze in der Organisationstheorie, Opladen

Mayring, Philipp (2003), Qualitative Inhaltsanalyse. Grundlagen und Techniken, 8. Aufl., Weinheim und Basel

Meyer, Rüdiger (2003), Wissensmanagement ist mehr als ein Archiv. Erfolgsfaktoren im Öffentlichen Sektor, in: Government Computing, 3. Jg., Nr. 11, S. 11

Milner, Eileen M. (2000), Managing Information and Knowledge in the Public Sector, London und New York

Nagel, Erik und Werner R. Müller (1999), New Public Management: (K)ein Wandel ohne Kulturentwicklung (!). WWZ-Forschungsbericht 1/99, Basel

Neubauer, Walter (2003), Organisationskultur, Stuttgart

Nohr, Holger (2001), Wissen wird zum Fokus betrieblichen Managements. Eine Kurzübersicht zum Wissensmanagement, Arbeitspapiere Wissensmanagement Nr. 4/2001, Stuttgart

Nohria, Nitin, William Joyce und Bruce Roberson (2003), What really works, in: Harvard Business Review, 81. Jg., Nr. 6, S. 43-52

Pan, Shan L. und Harry Scarbrough (1998), A Socio-Technical View of Knowledge-Sharing at Buckman Laboratories, in: Journal of Knowledge Management, 2. Jg., Nr. 1, S. 55-66

Pawlowsky, Peter (2002), Wozu Wissensmanagement?, in: Wissensmanagement. Zwischen Wissen und Nichtwissen, hrsg. von Georg Götz, 4. Aufl., Stuttgart, S. 109-126

Pemberton, Jonathan D. und Georg H. Stonehouse (2002), The Importance of Individual Knowledge in Developing the Knowledge-Centric Organisation, in: Knowledge Management in the SocioTechnical World, hrsg. von Elayne Coakes u.a., London u. a., S. 77-89

Pfeifer, Tilo u.a. (2001), Wahrnehmung und Realität in der Wissensmanagement-Praxis. Ergebnisse einer aktuellen Trendstudie zu den Problemlagen der Implementierung, in: new management, 70. Jg., Nr. 10, S. 28-36

Pieler, Dirk (2001), Neue Wege zur lernenden Organisation. Bildungsmanagement, Wissensmanagement, Change Management, Culture Management, Wiesbaden

Probst, Gilbert, Steffen Raub und Kai Romhardt (2003), Wissen managen. Wie Unternehmen ihre wertvollste Ressource optimal nutzen, 4. Aufl., Wiesbaden

Rosenstiel, Lutz von (2000), Wissensmanagement in Führungsstil und Unternehmenskultur, in: Wissensmanagement, hrsg. von Heinz Mandl und Gabi Reinmann-Rothmeier, München und Wien, S. 139-158

Ruggles, Rudy (1998), The State of the Notion: Knowledge Management in Practice, in: California Management Review, 40. Jg., Nr. 3, S. 80-89

Rüstmann, Marco und Jean-Paul Thommen (2002), Wissensmanagement, in: Management und Organisation. Konzepte, Instrumente, Umsetzung, hrsg. von Jean-Paul Thommen, Zürich, S. 445-481

Sackmann, Sonja A. (1991), Uncovering Culture in Organizations, in: Journal of Applied Behavioral Science, 27. Jg., Nr. 3, S. 295-317

Sackmann, Sonja A. (2002), Unternehmenskultur. Erkennen - Entwickeln - Verändern, Luchterhand

Sager, Michael und Markus Aebi (2003), Ganzheitliches Wissensmanagement bei der Credit Suisse Financial Services, in: zfo, 72. Jg., Nr. 2, S. 102-106

Sandkühler, Hans Jörg (2002), Natur und Wissenskulturen, Sorbonne-Vorlesungen über Pluralismus und Epistemologie, Stuttgart

Schein, Edgar H. (1992), Organizational Culture and Leadership, 2. Aufl., San Francisco u.a.

Schmelcher, Jill, Marion Witte und Richard Linxweiler (2002), Die unsichtbare Kraft. Mit gelebter Unternehmenskultur zum Erfolg, Wiesbaden

Schreier, Claus und Jean-Paul Thommen (2002), Interkulturelles Management, in: Management und Organisation. Konzepte - Instrumente - Umsetzung, hrsg. von Jean-Paul Thommen, Zürich, S. 403-444

Smircich, Linda (1983), Concepts of Culture and Organizational Analysis, in: Administrative Science Quarterly, 28. Jg., Nr. 28, S. 339-358

Sollberger, Bettina Anne (2004), Erfolgsfaktor Wissenskultur für ein ganzheitliches Wissensmanagement. Konzeptionelle Grundlagen und methodisches Vorgehen. Arbeitsbericht Nr. 74 des Instituts für Organisation und Personal der Universität Bern, Bern

Sollberger, Bettina Anne (2005), Wissenskultur als Erfolgsfaktor für ein ganzheitliches Wissensmanagement. Empirische Untersuchungen und Ergebnisse. Arbeitsbericht Nr. 78 des Instituts für Organisation und Personal der Universität Bern, Bern

Stenmark, Dick (2001), Leveraging Tacit Organisational Knowledge, in: Journal of Management Information Systems, 17. Jg., Nr. 3, S. 9-24 
Teschke, Klaus, Detlef Zimmer und Marc Diefenbruch (2002), Wissensmanagement erfolgreich einführen, in: Pallas Praxisberichte, o. Jg., S. 41-44

Thom, Norbert (1997a), Effizientes Innovationsmanagement in kleinen und mittleren Unternehmen. Grundkonzepte, praktische Instrumente und Wege zum Erfolg, Bern

Thom, Norbert (1997b), Management des Wandels, Grundelemente für ein differenziertes und integriertes „Change Management“, in: Die Unternehmung, 51. Jg., Nr. 3, S. 201-214

Thom, Norbert (2002), Einführung in die Führungslehre, 6. Aufl., Bern

Thom, Norbert (2003), Viele Barrieren bis zur Wissensgesellschaft. Gastkommentar, in: Basler Zeitung, 161. Jg., Nr. 167, 21. Juli 2003, S. 11

Thom, Norbert und Joanna Harasymowicz-Birnbach (2003a), Wissensmanagement im privaten und öffentlichen Sektor. Wie Staat und Privatwirtschaft voneinander lernen - Versuch einer Synthese, in: Wissensmanagement im privaten und öffentlichen Sektor. Was können beide Sektoren voneinander lernen?, hrsg. von Norbert Thom und Joanna Harasymowicz-Birnbach, Zürich, S. 15-39

Thom, Norbert und Joanna Harasymowicz-Birnbach (2003b), Wissensmanagement: Was können Staat und Privatwirtschaft voneinander lernen?, in: Die Volkswirtschaft, 76. Jg., Nr. 7, S. 50-53

Thom, Norbert und Adrian Ritz (2004), Public Management. Innovative Konzepte zur Führung im öffentlichen Sektor, 2. Aufl., Wiesbaden

Unterreitmeier, Andreas und Florian Schwinghammer (2004), Die Operationalisierung von Unternehmenskultur Validierung eines Messinstruments, Heft 18 der Schriften zur Empirischen Forschung und Quantitativen Unternehmensplanung der Ludwig-Maximillians-Universität München, München

Wagner, Wolf-Rüdiger (1999), Kulturtechnik Multimedia. Die Technikignoranz der Medienpädagogik und die Notwendigkeit ihrer Neuorientierung. URL: http://www.medienpraktisch.de/amedienp/mp4-99/4-99wagn.htm, 10. Oktober 2005

Weber, Roberto A. und Colin F. Camerer (2003), Cultural Conflict and Merger Failure: An Experimental Approach, in: Management Science, 49. Jg., Nr. 4, S. 400-415

Wiig, Karl M. (2002), Knowledge management in public administration, in: Journal of Knowledge Management, 6. Jg., Nr. 3, S. 224-239 